Artículos 

Artículos

Publicación semestral. ISSN 1409-2522

Volumen 76 - Número 1

Revista de las artes Julio - Diciembre 2016

\title{
La danza como dispositivo de resistencia: una exploración desde Danza Universitaria
}

\author{
María José Bejarano
}

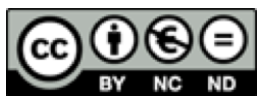

Esta obra está bajo una licencia Creative Commons

Reconocimiento-No comercial-Sin Obra Derivada 

Artículos

\title{
La danza como dispositivo de resistencia: una exploración desde Danza Universitaria
}

\author{
María José Bejarano' \\ Red de Investigación sobre el Cuerpo y las Corporalidades, Costa Rica \\ mariabeja@gmail.com
}

Recibido: 06 de julio de 2016 Aprobado: 15 de setiembre de 2016

\section{Resumen}

Se presenta una sistematización y revisión de los talleres de movimiento realizados por la Compañía de Danza Universitaria en el Recinto de Golfito de la Universidad de Costa Rica durante los años 20132014. Se utilizaron las técnicas cualitativas de entrevista, cuestionario y análisis de video. Los resultados muestran una intervención interesante que convocó el imaginario social de la comunidad, transgrediendo prohibiciones heredadas de la dinámica sociopolítica de la United Fruit Company, que ocupó este territorio durante el siglo XX. Se analiza la experiencia a la luz de conceptos de geografía humana, así como desde el enfoque de la antropología del cuerpo para proponer posibles alcances políticos de la danza como dispositivo de resistencia con raíz en las corporalidades.

Palabras clave: danza, corporalidad, poder.

\begin{abstract}
A systematization and revision of the movement workshops done by Danza Universitaria Company in the University of Costa Rica's seat in Golfito during 2013-2014 is described. The data was collected using qualitative techniques such as interview, questionnaire and video analysis. The results show an interesting intervention reaching the social imaginary of the community, breaking prohibitions inherited from the

${ }^{1}$ El presente artículo es un producto de una investigación realizada en colaboración para Danza Universitaria de la Universidad de Costa Rica, en el contexto del proyecto "Talleres de Movimiento en Comunidades".
\end{abstract}




\section{Artículos}

sociopolitical dynamics imposed by the presence of the United Fruit Company, which occupied this territory during the XX century. The experience is analyzed in the light human geography concepts, as well as body anthropology theory, to suggest the possible political significance of dance as a resistance device rooted in the corporalities.

Keywords: dance, corporality, power. 


\section{Introducción}

A partir del año 2006 la Universidad de Costa Rica establece su Recinto de Golfito -en lo que fueran previamente las instalaciones de la United Fruit Company (UFCo) -, y desarrolla en el sitio programas de docencia, educación y acción social. Entre ellos, grupos de representación deportivos y artísticos. Para el año 2013, se convoca a Danza Universitaria, Compañía de Danza Contemporánea de la Universidad, para dar capacitación al grupo de folklor de dicho recinto. Como parte de la línea de acción social de la Universidad -y de la Compañía- se propuso realizar talleres de movimiento, con la condición particular de que se abriera la convocatoria general a la comunidad de Golfito, y no solo estuviera abierta para los grupos de representación estudiantiles. La apertura tuvo como consecuencia un encuentro inesperado con el imaginario social heredado de los años de desarrollo de la UFCo, relacionado con un sistema de geografía humana organizado en función de esta compañía bananera. Este imaginario fue un emergente de la intervención, por lo que fue material a través del proceso de movimiento y creación. En este artículo se presenta una lectura de lo acontecido durante esta intervención en una red de relaciones entre la corporalidad, la institucionalidad, la cultura y la comunidad de Golfito.

\section{Golfito y la Compañía bananera}

Durante la tercera década del siglo XX, la
United Fruit Company trasladó sus operaciones del Caribe costarricense al Pacífico. La nueva administración de la UFCo se estableció en Golfito, una pequeña comunidad costera a la orilla del Golfo Dulce. Al llegar, la compañía absorbió las tierras de los productores de banano nacionales y de otros trabajadores de cultivos, obteniendo sus terrenos a través de contratos con el Estado costarricense, lo cual afectó de manera dramática la vida cotidiana de la comunidad (Jensen, 2012). Los contratos afectaron los intereses de los productores nacionales, además de transformar hasta la actualidad el imaginario político de ese contexto:

La presencia de la UFCo se tradujo en un ordenamiento territorial de toda la región acorde con los requerimientos productivos y administrativos, de la propia compañía; ordenamiento que se impuso sobre las unidades de administración política del Estado costarricense, las cuales eran de todas maneras apenas incipientes o limitadamente efectivas en esos lugares. De igual manera, se desarrolló una red vial y ferroviaria supeditada a la actividad de exportación y al transporte de la fruta hacia los barcos bananeros que atracaban en el muelle de Golfito y, al mismo tiempo, también se extendió un patrón de asentamientos humanos articulado funcionalmente con la dinámica de la compañía bananera. (Jensen, 2012, pp. 200-201)

El asentamiento de la UFCo en la comunidad de Golfito desembocó en una transformación de la geografía política de la zona, determinando así los espacios de manera 


\section{Artículos}

que le dieran soporte y desarrollo al enclave, mientras que los demás aspectos de la vida social fueron desplazados y puestos como prioridades secundarias. Este rasgo destaca incluso hasta hoy en el imaginario costarricense, en el cual Golfito refiere preponderantemente a su zona de libre comercio. Esta transformación económica y política fue clasificatoria de los grupos sociales:

La reorganización del espacio en Golfito revela la jerarquización del espacio, la cual dividió a la ciudadela en tres zonas: la zona blanca o americana, la zona amarilla o administrativa y la zona gris $u$ obrera. La zona americana albergaba las viviendas de los funcionarios de alto nivel y sus instalaciones recreativas, entre ellas piscina, canchas de tenis y campo de golf, además de amplios jardines. La zona amarilla contenía las viviendas de funcionarios de nivel intermedio y de empleados solteros; los edificios administrativos y el club social de la comunidad de empleados superiores o medios de la compañía; además, servicios de salud y de proveeduría. La zona gris correspondía a los trabajadores portuarios, la capitanía del puerto y la infraestructura operativa. (Jensen, 2012, p. 204)

Estos cambios en infraestructura determinaron de alguna manera la lectura interna de quiénes eran las personas, qué relaciones tenían, qué acceso tenían a una diversidad de actividades y a qué podían o no aspirar dependiendo de la ubicación de su residencia. Esta asimetría en la vivencia del espacio en la zona ha sido trabajada por Jensen (2012) con mucha más profundidad y vinculada con el concepto de sintaxis espacial. Para este autor, esta tiene las siguientes implicaciones:

Estas zonas están compuestas por elementos entre los cuales existen relaciones -implícitas y explícitas- sujetas a una sintaxis espacial, o sea a un conjunto de reglas que señalan la dirección de los flujos comunicativos y de tránsito, el acceso a los componentes del espacio (y, por lo tanto, la exclusión o la segregación con respecto a esos componentes). Asimismo, se refieren a la coordinación entre los niveles de autoridad y la utilización del espacio. En otras palabras, la sintaxis espacial cumple una función clasificatoria de las actividades sociales y, al mismo tiempo, representa un mecanismo que articula las diferentes dimensiones socioespaciales de una estructura urbana. (p. 205)

Concluye el autor que, en este fenómeno de intereses económicos, hay inevitablemente consecuencias sociales en los vínculos como grupo comunitario y, de este modo, una experiencia de la subjetividad individual. Existe una relación directa entre la distribución del espacio y la experiencia vital de los sujetos. De esta manera:

Las cosas y las personas reciben una localización; el espacio se transforma en un lugar que cada quien ocupa en el entramado social y esa posición constituye una testificación del significado de sus acciones en los procesos de producción y reproducción social. El lugar es el significante de las personas y las cosas. De esta manera, el orden social presupone un espacio y a la vez lo crea y transforma. 
(Jensen, 2012, p. 205)

En este fenómeno de clasificación espacial en el microsistema económico de Golfito, se podría leer el concepto de heterotopías del poder de Foucault, las cuales tienen que ver con el establecimiento de espacios que determinan la vivencia y el intercambio social que, de alguna manera, fomentan la organización del espacio en función del ordenamiento y el control. Tal como afirma Rodríguez (2006):

El poder se articula directamente sobre un tiempo y un espacio, asegura su control y garantiza sus usos. Así, las restricciones espaciales no hacen otra cosa que revelar estructuras de poder y los mecanismos de segregación utilizados, para ello traducen las relaciones de poder implicadas en la construcción de identidades y alteridades entre la ciudadanía. [...] Otras veces son lugares perfectamente visibles y cartografiables, pero sometidos a un canon de conducta propio que los convierte en instrumentos de fijación a un determinado aparato de normalización de las personas. (p. 171)

El orden espacial y la disposición de las zonas permitidas para cada grupo social determinan la experiencia personal, llegando a controlar a las personas y permear sus vidas de un discurso de poder que llegan a encarnar. Desde este punto de vista, se puede afirmar que la clasificación social es una clasificación de los cuerpos, que desde un régimen de autoridad definen cuáles cuerpos importan (ver Butler) y cuáles no. Es decir, cuáles cuerpos (cuáles vidas) merecen una existencia con dignidad y cuáles están exclusivamente al servicio de producción de riqueza. En este contexto el poder atraviesa los cuerpos de esta manera: no precisamente en una consigna vertical, sino a través discursos, distribución del espacio y del tiempo. Afirma Macías (2009):

Desde el acatamiento de prácticas que definen actividades, métodos de gestión del espacio y cálculos del tiempo y del movimiento, se espera forjar un cuerpo productivo, sometido a relaciones de fuerzas irreductibles a la violencia. Para Foucault, esta serie de acciones, actos que incitan, inducen, desvían, facilitan «no son simplemente un arte de distribuir cuerpos, de extraer de ellos y de acumular tiempo, sino de componer una fuerza para obtener un aparato eficaz» que hacen de las iniciativas del cuerpo, una lista de actividades rutinarias, controladas y seleccionadas, en donde se definen y delimitan las maneras de ver y organizar la vida. (p. 98)

Desde este punto de vista, en el microsistema descrito de Golfito -así como en la sociedad en general de una manera menos evidentese vislumbra un bosquejo espacial que impone las posibilidades a las que se pueden aspirar y que además se asumen como una verdad y se encarnan en las personas.

Macías (2009) interpreta el control de los cuerpos como una necesidad de contener las fuerzas excesivas que emanan de ellos. Es por esto que los cuerpos son blancos de control, porque poseen la fuerza necesaria 


\section{Artículos}

para crear una maquinaria poderosa de producción y riqueza. Sin embargo, el control de los discursos de poder sobre el cuerpo no es unidireccional, estos tienen también la posibilidad de irrumpir en un sistema, aun cuando exista tanta fuerza sobre ellos, siempre exceden en algo al control y son capaces de resignificar las posibilidades, los espacios y transformar su entorno.

Llegamos al punto en que, sabiendo que en todo momento hay técnicas y procedimientos que el individuo aplica sobre él mismo y sobre los otros, éstos no son sólo tecnologías en términos de obediencia, sino que también pueden ser de resistencia. Resistencias que se mueven dentro de las relaciones de poder, que son inestables y permiten estrategias para modificarlas, o, al menos, para enunciarlas como posibilidad. (Macías, 2009, p. 44)

Es en los espacios de creación de realidades, en la imaginación de otros escenarios posibles donde se cuela la libertad del ser humano común, dominado en su cotidianidad por el poder de las estructuras e instituciones sociales. Desde ahí se propone al arte, y la danza en particular, como un dispositivo de resistencia que, en su íntimo vínculo con la vivencia de contrapuestos -como son lo individual/colectivo, lo personal/político-, incita a una movilización hacia otras formas de vincularse con otros, de repensar la propia historia y de cuestionar las posibilidades que ofrece el espacio.

Si el accionar de la danza apela a un encuentro con el mundo y con otras corporalidades, si impulsa un movimiento de fuerzas desde lo táctil que problematiza lo real en el escenario y en los espacios que ha decidido reconfigurar desde la construcción de otras relaciones y geografías de acción, entonces, la danza habita ese cruce entre el arte y lo político. (Macías, 2009, p. 130)

A continuación, se analiza un proceso de intervención desde la danza en la comunidad de Golfito, a la luz de estos conceptos, problematizando la experiencia de la danza en un espacio cartografiado por el poder y proponiendo una lectura de resistencia de los cuerpos danzantes.

\section{Metodología}

La intervención de Danza Universitaria en la comunidad de Golfito consistió en 12 talleres de movimiento abiertos al público; un proceso similar se replicó durante dos años consecutivos: 2013 y 2014. Los participantes fueron en total 24 personas entre los 9 y los 33 años de edad. El cierre del segundo proceso se realizó con una presentación de la muestra final de lo trabajado, con la participación de más de 400 personas de Golfito y comunidades aledañas. Cada taller consistió en un calentamiento, luego del cual se dieron consignas para explorar el movimiento de forma individual, en parejas o en grupos. Esto también implicó ejercicios de improvisación en los que las personas participantes propusieron movimiento, de los cuales se tomó el material para la muestra final. Hubo un coordinador principal que facilitó 
la mayoría de talleres, pero en algunas ocasiones se invitó a otros miembros de la Compañía de Danza Universitaria a colaborar en la facilitación. En total participaron 4 miembros de la Compañía en la facilitación de los talleres.

Para el presente año se trabajó en la sistematización del proceso de inserción en la comunidad de la Compañía, lo cual implicó partir de varias fuentes de información que dieran cuenta de lo sucedido dos años después de terminado el segundo proceso. Para esta investigación se recurrió a tres fuentes:

1. Análisis del registro en video. Durante los talleres de movimiento se grabaron videos con el fin de sistematizar muestras de lo realizado. Estos videos contenían fragmentos de las sesiones y no las sesiones completas. Sin embargo, se consideraron datos importantes para analizar la intervención, por lo que se incluyeron en la presente investigación la mayor cantidad de videos cortos existentes en los archivos de Danza Universitaria. Se analizaron en total 46 minutos de video, los cuales fueron asumidos como representativos del proceso total, en el sentido de que mostraban la estructura del taller. Algunas dudas sobre las dinámicas observadas se aclararon con el facilitador del proceso. Se construyó una guía de observación que permitía considerar los siguientes puntos: (1) la interacción entre participantes, de facilitador a participantes y de participantes a facilitador; (2) cuál era el origen del movimiento (imitación del facilitador; invitación al movimiento a través de la palabra; invitación al movimiento a través del contacto físico y/o exploración propia de movimiento); (3) quién utilizaba la palabra entre participantes y facilitadores y (4) la distancia que se observaba entre las personas involucradas en la sesión. Los videos fueron analizados por parte de dos observadores distintos, en días y lugares distintos y finalmente se contrastaron las impresiones. Las observaciones coincidían aproximadamente en un $90 \%$ de los casos y se buscó el acuerdo en los casos en los que no se encontró de primera entrada, a través de una discusión entre observadores.

2. Entrevista semi-estructurada. Se realizó una entrevista al coordinador de los Talleres de Movimiento en Golfito. El fin de la entrevista fue indagar en los objetivos de la intervención, en los resultados obtenidos desde el punto de vista del creador y los retos que se pudieron sobrellevar durante el proceso de inserción en la comunidad, así como recabar testimonios surgidos de la experiencia. De esta manera, se pretendía completar la información referente al proceso de los talleres de movimiento realizados, contrastándola con la observación de los videos y los cuestionarios enviados a las participantes.

3. Cuestionario: Se envió un cuestionario por correo electrónico a 7 de las participantes del taller para ser completado 


\section{Artículos}

en línea. A estas personas se les contactó telefónicamente para solicitarles su participación en la investigación y accedieron a colaborar. Sin embargo, solamente 3 personas completaron el instrumento de manera muy escueta. Por lo que la información brindada no aportó para los resultados descritos aquí.

Una vez obtenidos los instrumentos y recogidos los datos, se procedió a sistematizar la información que resultó de la exploración realizada. Luego se procedió a la contrastación y análisis de los datos.

\section{Resultados}

Los contextos culturales en los que vinieron a insertarse estos talleres son determinantes para los resultados que tuvieron en la comunidad, a través de los participantes. El relato del facilitador da cuenta de la vivencia de sí mismo como creador, tanto como lo que percibe del proceso de los participantes a través de testimonios que ellos y ellas mismas le dan, las cuales se refieren a imaginarios que componían la esfera social y que fueron cuestionados por la práctica de la danza/ movimiento. Por su parte, los videos aportan para conocer cuál fue la ruta práctica en el desarrollo de los talleres de movimiento. A continuación, se desarrollan estos aspectos.

\section{La estratificación social de la UFCo}

El contexto político, económico y social más preponderante observado en la comunidad se relaciona con la huella que dejó la inserción de la bananera, aún muy fuerte incluso en las personas más jóvenes que participaron en los talleres. Abrir las puertas del taller a la comunidad, y trabajar en el Club Centro (centro social de la clase privilegiada durante los años de la UFCo), representó un cuestionamiento a la sintaxis del espacio plasmada por la presencia de la compañía en esta comunidad costera. Tal como lo narra el coordinador durante la entrevista:

Tradicionalmente había una diferencia entre la zona donde están todas las edificaciones de la bananera de lo que es el pueblo civil, que así le dicen ellos. Ya con sólo el nombre uno dice que hay algo ahí extraño... La gente de la bananera eran los privilegiados, la clase alta, todas las instalaciones y las edificaciones son super bonitas, hay unas casas preciosas, todo eso pasó a ser parte de la Universidad. Creo que quedó por ahí en el imaginario, entonces no se acercan, digamos, no sienten que es accesible. (comunicación personal)

La apertura de los talleres a la comunidad fue un planteamiento del coordinador, basado en un compromiso social de Danza Universitaria de dar acceso a las comunidades rurales a la práctica y apreciación de la danza. Sin embargo, hasta llegar a la zona, se identificó esta particularidad política que representaba el espacio físico de la universidad para la comunidad. Se percibía una suerte de restricción heredada para entrar al territorio de la universidad, restricción que no se concretaba en 
la realidad de la institución, ya que el espacio se plantea abierto a todas las personas.

Como vemos, el acceso a actividades educativas y culturales reservado para las clases privilegiadas es una sintaxis espacial que pareciera haber permeado a la institución que representa la universidad, al colocarse en el espacio ocupado anteriormente por la UFCo. La institución educativa se inserta en este imaginario, pasando a contemplarse como un privilegio para las clases altas, para las personas que ocupan cargos poderosos y de autoridad en la comunidad. Aparentemente, este imaginario no había sido revertido por la Universidad con anterioridad a los talleres, por lo que al llegar no se encontró vínculo entre esta y la comunidad de Golfito. Dice el coordinador de los talleres:

Los estudiantes de secundaria, por lo menos los que yo conocí, ellos nunca habían entrado a la U, ni se imaginaban llegar ahí. Entonces eso fue interesante porque sí se rompió a través de estos talleres que no se acercaran. De hecho, yo pedí por favor que se hicieran los talleres dentro de la $U$ porque es necesario llevar a la gente ahí. (M.B., comunicación personal)

Una vez transgredida dicha restricción heredada, la segunda ruptura radicó en que los grupos que participaron en los talleres procedían de diversos estratos sociales. Había personas de colegios públicos y de colegios privados. En este sentido, se agregó un segundo movimiento, promoviendo una aproximación entre grupos sociales clasificados como incompatibles, y distanciados unos de otros, aún años después de establecida esta división geográfica y localización política.

Entonces había un grupo como de 5 o 6 que eran gimnastas, pero son de otra clase social, de colegios privados y de otra zona totalmente entonces era muy interesante que había unas chiquillas así todas técnicas que habían recibido toda una preparación y gimnasia y todo. Y por otro lado estos de colegio público que en la vida se habían comunicado, que además era una separación entre colegio público y los colegios privados de Río Claro. Y ahí se revolvió el asunto. (comunicación personal)

Esta separación del grupo, basada en lo económico, fue diluida durante el taller, permitiendo que se acercaran los grupos sin importar su procedencia, que pudiesen compartir desde el cuerpo, escucharse, crear en conjunto, devolviéndoles la posibilidad de ser comunidad. Aún cuando muchas de las razones para no compartir son sociohistóricas, la sintaxis espacial ha tenido un rol profundo en esta separación, la cual se trabajó a través del cuerpo entre los grupos participantes del taller.

Por las mismas dinámicas del taller sin que ellos se dieran cuenta, se estaban revolviendo, y yo procuraba mezclar los grupos, mezclar a la gente con otro objetivo sin que ellos tuvieran que hablar mucho, porque en realidad en los talleres no se habla casi, pero sí se trabaja mucho corporalmente, sí hay contacto físico y sí tienen que trabajar en equipo. Entonces 


\section{Artículos}

eso fue como romper un poco, porque yo creo que con la palabra se conduce mucho a de qué clase social soy[...](comunicación personal)

Es así como se encuentra un cuestionamiento sobre la creencia de que la danza es un privilegio para las clases altas. Se descoloca la danza, la cual pasa de ser percibida como exclusiva de las personas con más recursos económicos, para ser una herramienta de reflexión desde la corporalidad y el movimiento que todas las personas pueden aprovechar en su derecho a la cultura, además de un insumo para mezclarse entre grupos de diversa procedencia y condiciones sociales.

Esta mezcla también se dio con personas de diversas edades y generaciones. Aunque participaron en su mayoría adolescentes, el proceso abierto permitió incorporar adultas y una niña de nueve años. Esto dejó que las generaciones se influyeran entre sí, con la particularidad que trae cada etapa del desarrollo. Lo anterior enriqueció la vivencia del taller. Por ejemplo, dice el coordinador:

El hecho de que hubiera una niña en ese primer taller, que hubiera una niña que además era súper libre y súper conectada con el taller, provocaba una admiración en todos los demás, yo siempre la usaba como inspiración, yo decía "vean qué belleza como uno en la niñez puede jugar más libremente" y todos lo podían ver en ella, entonces era una influencia muy positiva para los que estaban más reprimidos. (comunicación personal)

La mezcla generacional podría resultar, en algunos casos negativa; sin embargo, al resaltar el aporte que cada participante daba al grupo, se convirtió en una experiencia amena, en la que las personas estaban aprendiendo de las demás, sumado a que se sentían en libertad para aportar desde sí mismas lo que desearan.

Entonces una vez que eso se genera, creo que el proceso para ellos fue muy rico en el sentido de que se les brindó o habían encontrado un espacio donde podían expresarse corporalmente o expresarse de otra manera distinta a la otra que tal vez es más racional o que tienen una calificación los adolescentes, por ejemplo, a ellos se les decía «aquí vamos a eliminar los conceptos de bueno/malo, las calificaciones, nadie se va a quedar ni va a ganar un premio ni nada de eso. Vamos a eliminar todas esas presiones». Entonces una vez que la gente ve que de verdad es así, y que no se les está regañando, ni demandando, ni obligando a nada... Yo creo que ellos en su proceso empiezan a disfrutar y el expresarse en un espacio que se los permite. (comunicación personal)

El movimiento generó así un espacio necesario para las personas en el que se logró inscribir el cuerpo en el movimiento; la dinámica social y la historia personal en el cuerpo; lograr que las personas se preguntaran qué era su cuerpo, qué experiencias guardaba y cómo las expresaban en sus relaciones sociales en la comunidad. Resaltar del cuerpo la vivencia personal le permitió a cada participante adquirir una nueva visión de sí misma, contemplarse en aspectos diferentes de 
los que había explorado hasta el momento y saltar restricciones personales, sociales y políticas que permeaban su experiencia corporal. Ejemplos de estos saltos son los que se narran a continuación:

Hay un caso muy interesante de una señora que su pareja, su esposo, llegaba ahí a ver el taller por ratos y la veía por la ventana y todo. Y después ella me comentaba que después su esposo... o sea que se había erotizado mucho la relación. Porque ella se movía, llegaba siempre sudada y todo y él viéndola por una ventana... Como que algo se estimuló en ellos en ese sentido. Decía "es que ahora mi esposo me ve... está todo erotizado conmigo todo el tiempo desde que me ve en este taller". Y ella se sentía mucho más erótica, más sensual, porque también había retomado un poco el contacto con su cuerpo.

Hay un muchacho que empezó a demostrar sus capacidades como líder, y entonces yo lo veía que él estimulaba y jalaba mucho a sus compañeros, y los llevaba era como muy... esas habilidades de liderazgo. Pero además él, su personalidad, desde lo que yo observo era que empezó muy retraído y de pronto se empezó como a empoderar, seguro le pegó por la autoestima o su relación con el cuerpo y se volvió un chico como súper sexy. Entonces todas las amigas estaban como encima de él... (comunicación personal)

A través de la reflexión desde el cuerpo se da la oportunidad a las personas de ampliar sus lenguajes cotidianos, para sumar el lenguaje corporal, aprendido desde sus propias dinámicas, desafiando sus propios contextos y elaborando a través del movimiento sus experiencias de vida para dar pie a otras inquietudes e iniciativas. Los talleres implicaron la puesta a disposición de un tipo de reflexión no precisamente atravesado por la palabra, y que sin embargo tiene consecuencias visibles en la vida de las personas, como las que se narraron anteriormente.

\section{Análisis de los videos}

Los fragmentos observados son en total 24, que se sitúan en diferentes momentos de la intervención de los años 2013 y 2014 . No se analizan entonces como un proceso, pero sí se puede observar que hay una secuencia en la manera en que se interviene, debido a que se propuso la misma estructura en ambos años y se ordenaron por fecha de grabación. En ese sentido, se analiza aquí el modelo de intervención, con las propuestas que realiza el facilitador y la reacción que se observa en las y los participantes.

Se observa en los primeros videos que la palabra acompaña el movimiento casi en todo momento. El facilitador describe el movimiento mientras lo realiza y guía a las personas, como si estuviese llevándolos en una visualización de su propio cuerpo. Esto se visualiza durante los primeros seis videos. Las personas participantes tienen una posición pasiva, están al tanto de las instrucciones, imitando el movimiento, pendientes del cuerpo del facilitador. Mientras tanto, el 


\section{Artículos}

\section{Fotograma 1}

Hasta el sexto video se observa imitación hacia la facilitadora, en una especie de espejo. El grupo se espeja en la facilitadora y esta guía el movimiento con el cuerpo y a través de la palabra

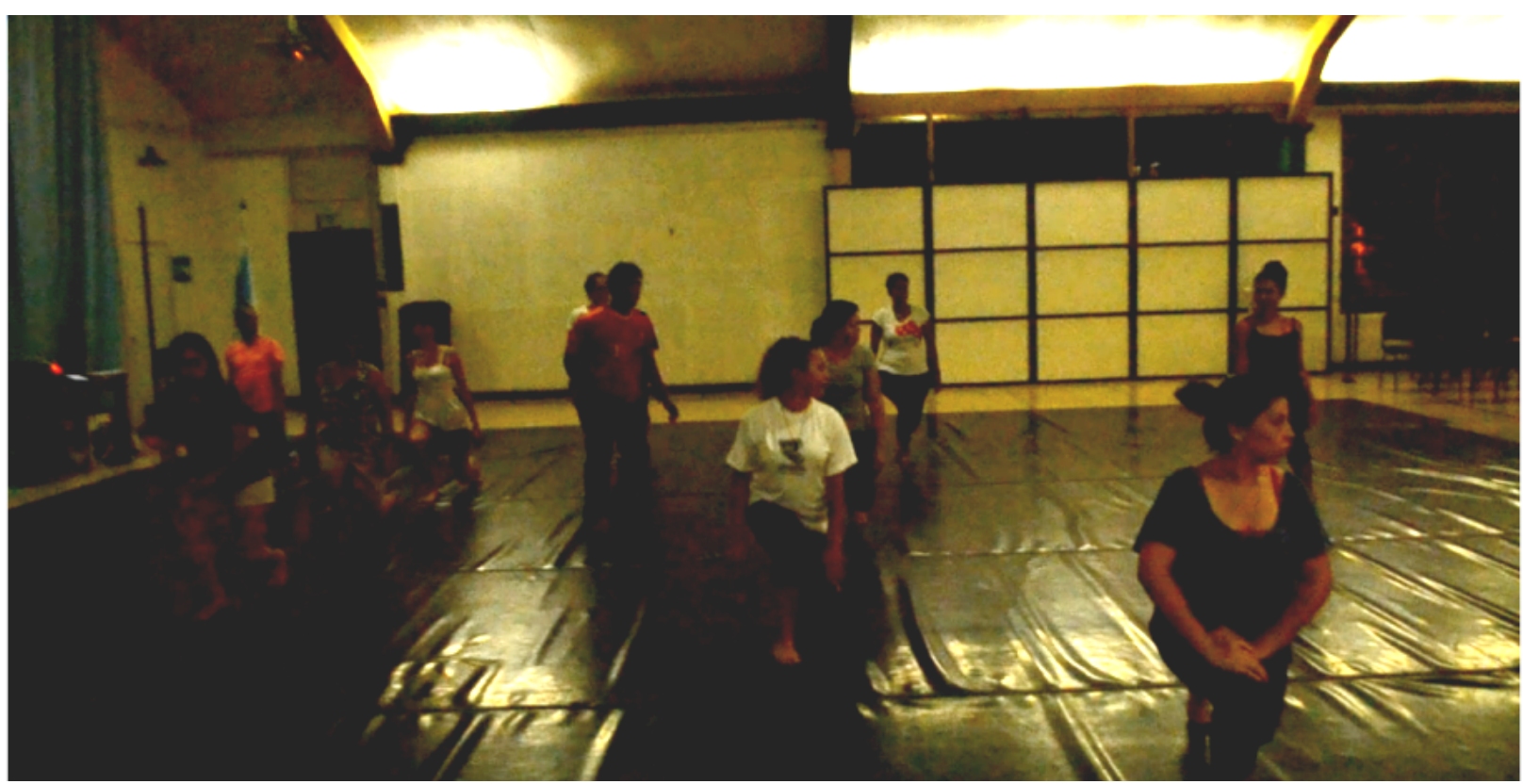

Fuente: Archivo Danza Universitaria

facilitador está también muy presente en su propio cuerpo, mostrando de la manera más clara los movimientos, y mientras tanto los describe con palabras. También guía al grupo en el ritmo, a través de cuentas que marcan el paso con respecto a la música o tempo.

Para el séptimo video se visualiza por primera vez que la facilitadora deja de mostrar algunos pasos con su cuerpo y les indica a los participantes que los realicen. Aquí, entonces, el grupo deja de espejarse en la facilitadora y, por primera vez, se presenta el reto de buscar en sus cuerpos el movimiento guiado anteriormente. Aún en este punto, no hay interacción entre las y los participantes, ni hay preguntas o invitaciones de ningún tipo hacia la facilitadora. La dinámica es unidireccional, desde la facilitadora que da la instrucción y el grupo que la sigue. La propuesta de movimiento sigue siendo desde la facilitación.

Seguidamente, a partir del décimo video, hay más libertad de movimiento, el grupo está más activo aunque aún no se le da la oportunidad de proponer mucho movimiento. Todavía la interacción sigue teniendo una dirección de facilitador a participantes. La diferencia radica en que las personas 


\section{Fotograma 2}

Las personas comienzan a proponer movimiento desde secuencias básicas como la caminata. La mirada empieza a llevarse hacia los otros participantes.

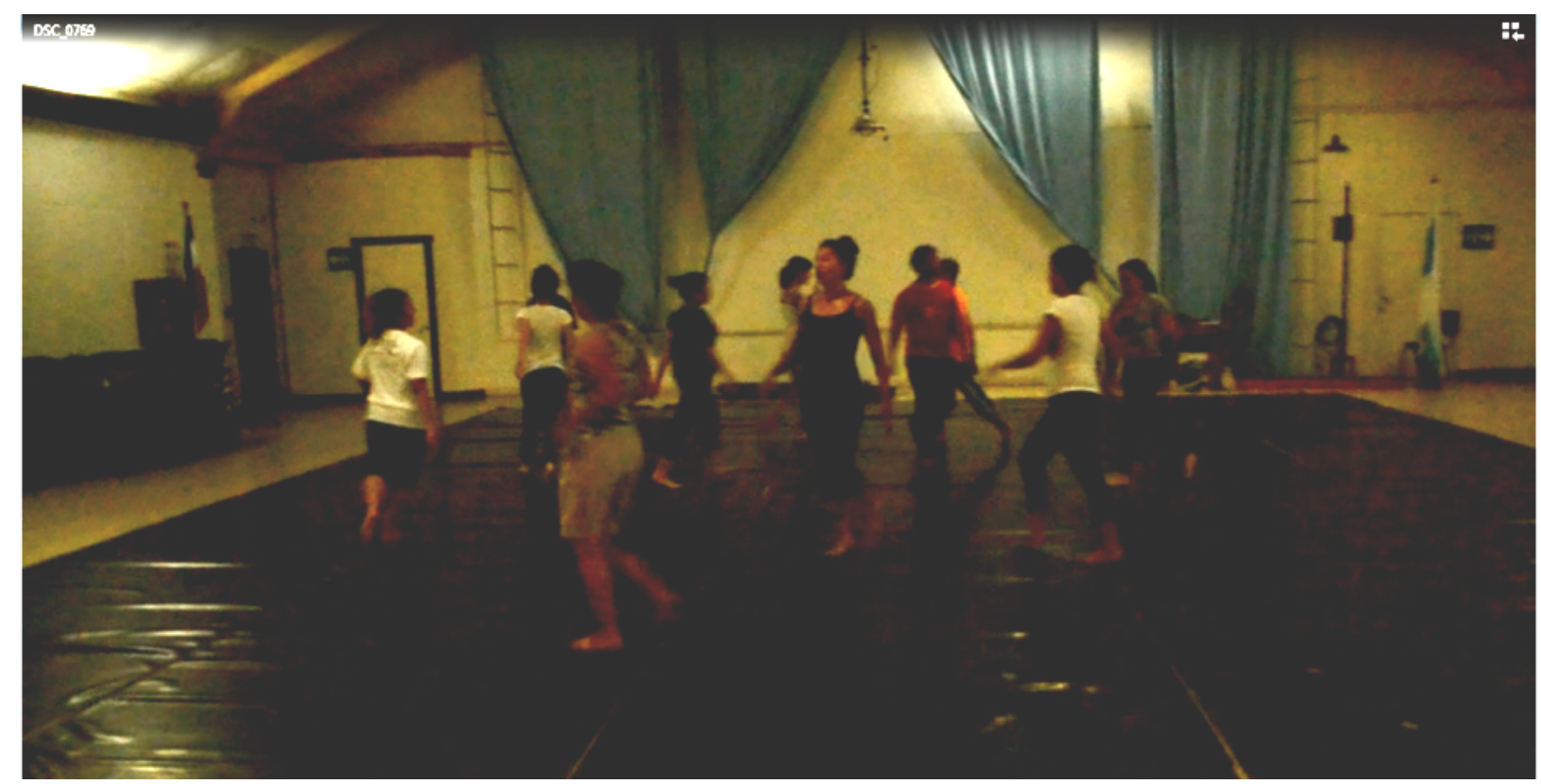

Fuente: Archivo Danza Universitaria

evidencian más comodidad a través de gestos, la fluidez del movimiento y la velocidad con el que los realizan.

Llama la atención que durante el video número doce, se observa una disminución significativa en el uso de la palabra y la dinámica del taller ya no está centrada en la imitación del movimiento del facilitador, sino que inicia la exploración del movimiento personal. Es decir, ya la consigna no es seguir el movimiento del facilitador; tampoco es hacer un movimiento aprendido sin la guía del facilitador, sino que el reto escala a una búsqueda de movimiento propio; no hay un guion, sino que la persona debe proponer desde su propio cuerpo. El grupo se ve más fluido a la hora de improvisar o descubrir movimientos por su propia cuenta. Se puede leer en estos aspectos que el grupo está consolidándose, ya conoce la dinámica del taller y va tomando confianza. Esta confianza implica libertad para proponer movimiento, un salto hacia un cuestionamiento desde el cuerpo de las cosas que les son más familiares, sus costumbres encarnadas.

La palabra ya no describe un movimiento a seguir, ya no es un guion de alguna manera estructurado, sino una invitación, una 


\section{Fotograma 3}

El grupo explora movimiento no estructurado, libremente en el espacio. Primer indicio de la mirada interna, hacia el propio movimiento.

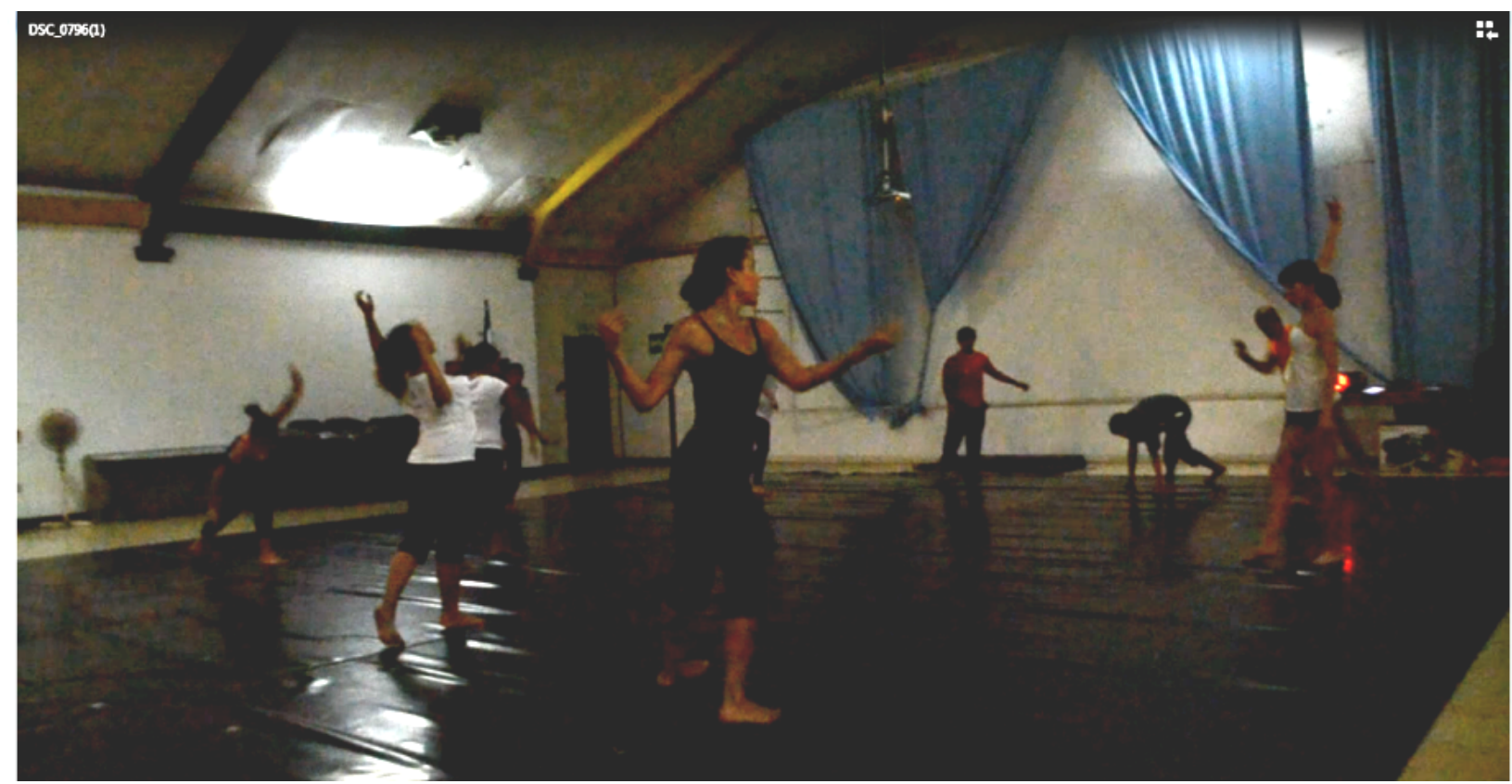

Fuente: Archivo Danza Universitaria

propuesta para que las personas ubiquen en sus cuerpos diversos sentires que les den la posibilidad de crear movimiento. Se escuchan consignas como: "busque la torsión", "encuentre esta sensación.... La mirada ya no se centra en la facilitadora, sino que pasa por la observación de otros participantes para llegar en este punto a ser una mirada interna, una conciencia de sus movimientos. Aún las y los participantes no asumen la palabra, pero sí comienzan un decir desde sus propios cuerpos.

El video número trece introduce el trabajo en parejas. El facilitador y co-facilitadora muestran cómo guiarse el uno al otro. En este momento, el foco de la mirada y el movimiento cambia de vía, de un participante observando un facilitador; pasando por un participante observándose a sí mismo para llegar a un participante observándose a sí mismo mientras vive la invitación de lo que otro(a) participante ha podido observar en sí mismo también. Se rescata que, con el paso del tiempo, las parejas se apropian mayormente del espacio y del cuerpo, mostrando más expresividad y espontaneidad en los movimientos. El lenguaje corporal empieza 


\section{Fotograma 4:}

Las personas exploran el movimiento en parejas en una relación dialéctica entre la conciencia del propio movimiento y la conciencia del otro participante.

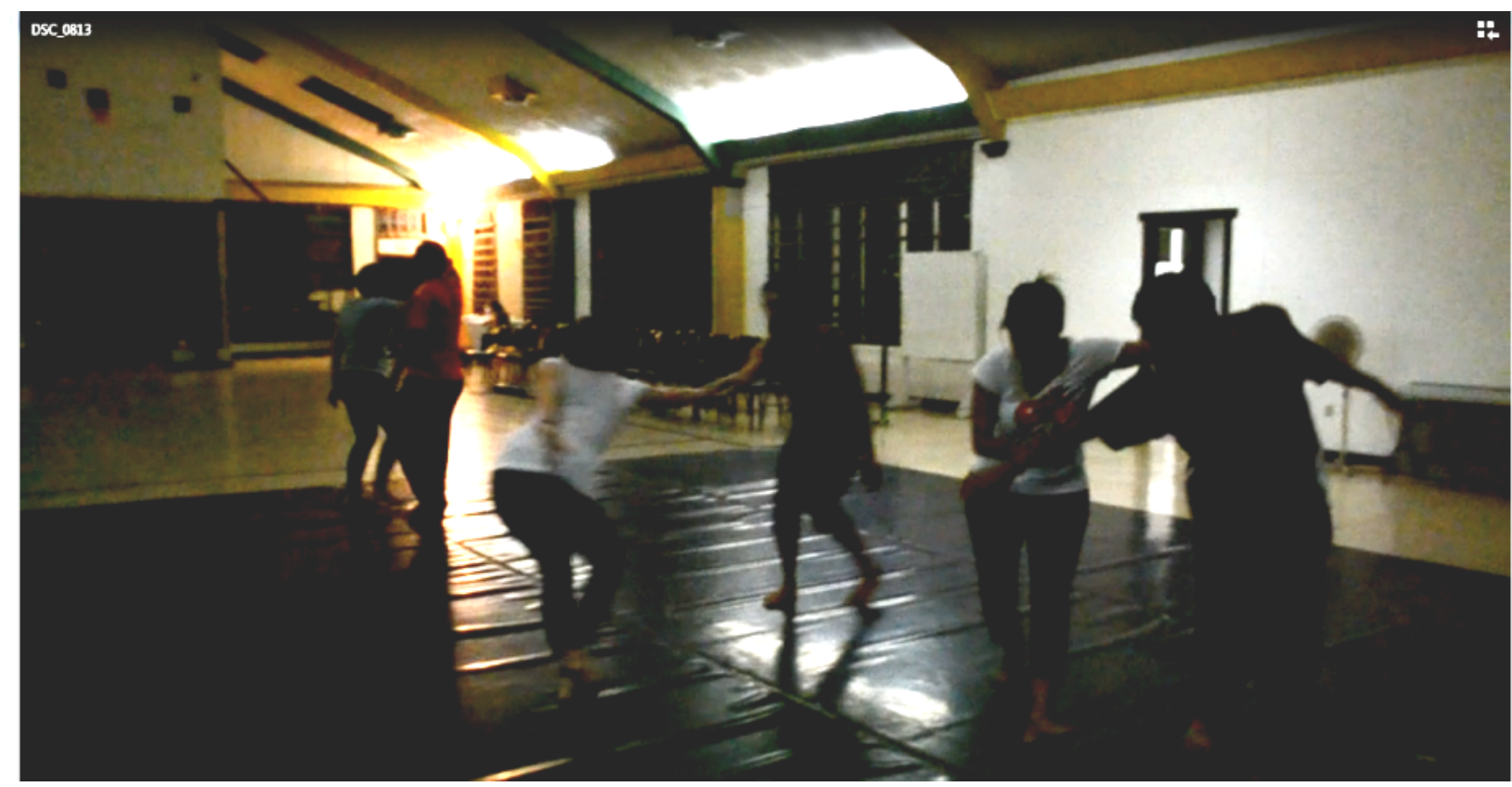

Fuente: Archivo Danza Universitaria

a tomar forma en ellas y ellos mismos, y comparten entre sí los mensajes que han ido logrando producir.

En este punto también se observa lo que después el facilitador aclara en entrevista que es parte de la metodología: cada semana entra un o una facilitador(a) distinto(a) a realizar el calentamiento. En la entrevista, el (la) facilitador(a) afirma que esto después no parecía tan buena idea, porque algunas veces no les gustaba o comparaban entre sí a las distintas personas que dirigían los calentamientos. Podría ser que, en este descubrimiento del propio cuerpo, hubiese una dificultad para adaptarse a un nuevo guía, que con un lenguaje del cuerpo distinto iniciara una nueva travesía. Es posible, asimismo, que los participantes sintieran un retroceso con respecto a lo que habían avanzado, porque los códigos, los mensajes que transmitían estos nuevos lenguajes, se sentían como volver a empezar la exploración del cuerpo. Esto queda como una inquietud para futuras intervenciones. La danza, como todo lenguaje, es un código que se aprende en el otro y cada persona que guía hace reconocer en el propio cuerpo facetas distintas. Toma tiempo desarrollar 
Artículos

\section{Fotograma 5}

Las personas participantes improvisan con movimiento propio.

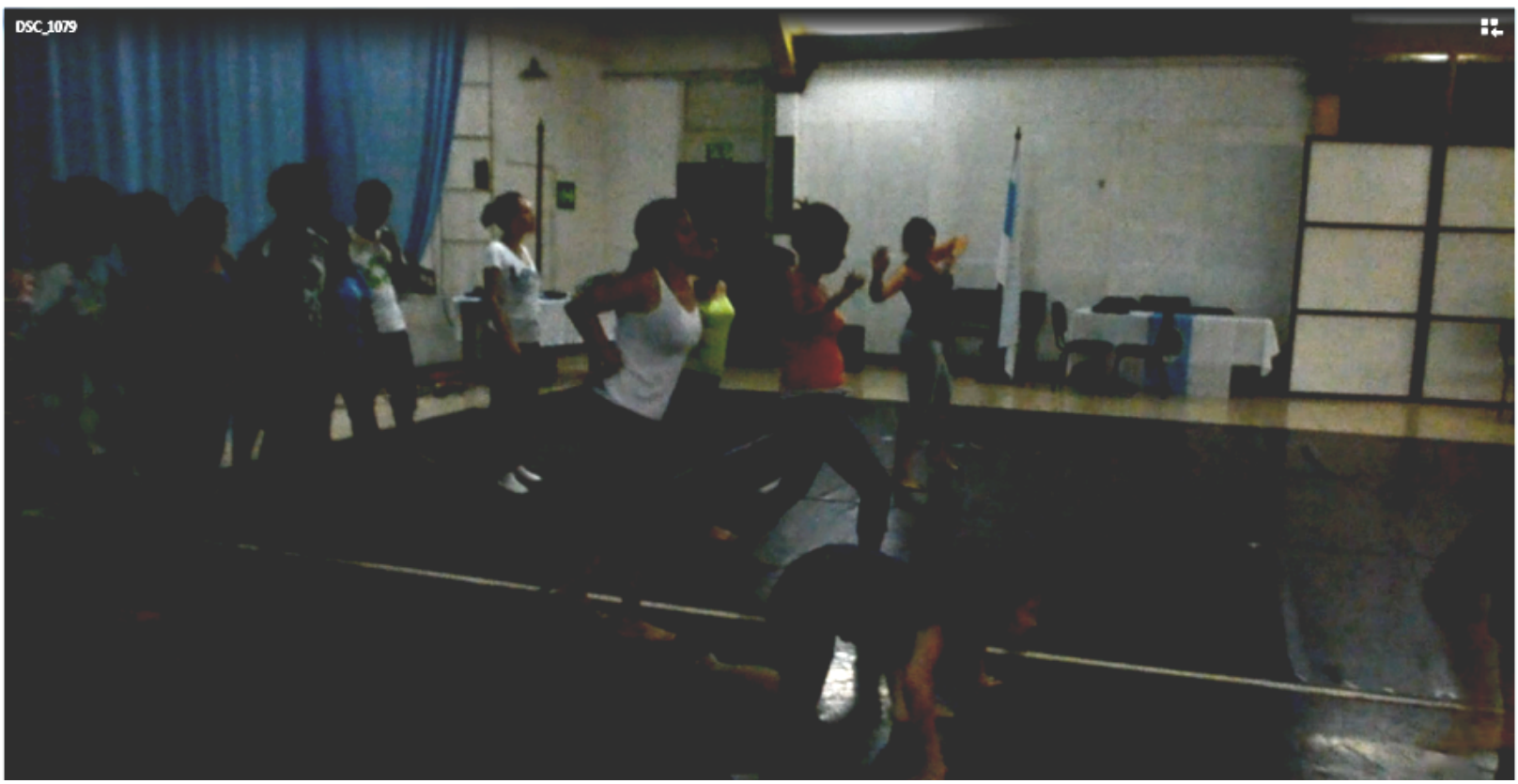

Fuente: Archivo Danza Universitaria

la capacidad de integrar las distintas facetas que otro va mostrando en uno mismo.

Al final de la observación, se ve a las y los participantes trasladándose de un extremo al otro del salón realizando movimientos con el cuerpo de forma libre, en conjunción con la música. Van pasando de uno en uno, y cada persona explora el movimiento con libertad. Algunos se permiten movimientos más fuertes y atrevidos que otros. Sin embargo, pareciera que disfrutan del ejercicio en la expresión de sus rostros. La facilitadora da palmadas cada cierto tiempo para movilizar la dinámica grupal. Una vez que llegaron al extremo del salón continuaron bailando. Durante el último video las personas que en otros videos se observan un poco más cohibidas, presentan mayor expresividad y apropiación de su propio cuerpo y del espacio.

Para esta fase ya casi no hay intervención a través de la palabra, tampoco el facilitador es el origen del movimiento, sino que las personas se atreven a crear movimiento propio. Se ve interacción entre las y los participantes; hay preguntas y comentarios hacia el facilitador y se desarrolla más contacto físico. La dinámica vertical que 


\section{Fotograma 6}

Las personas participantes improvisan con movimiento propio.

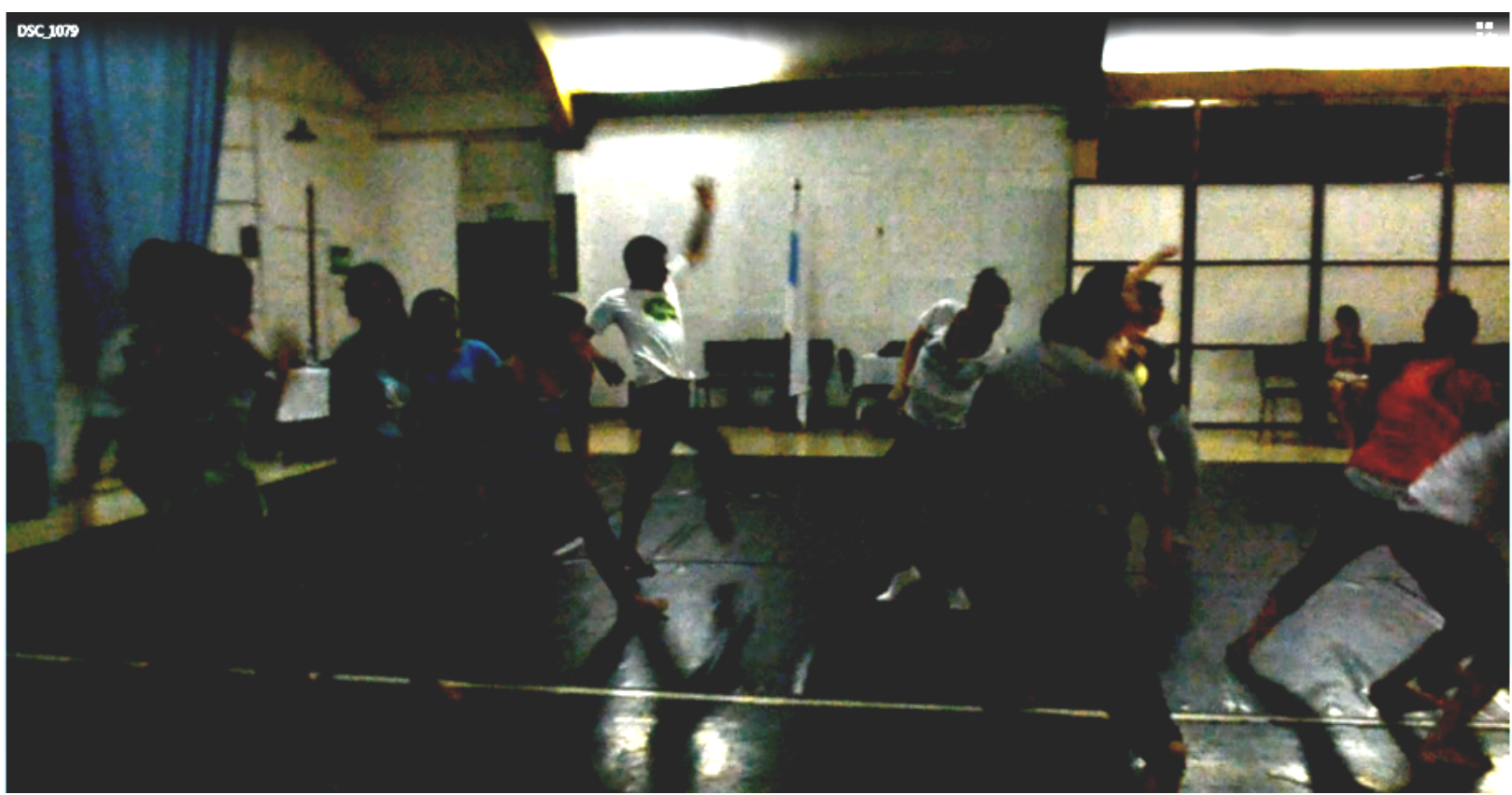

Fuente: Archivo Danza Universitaria

se observaba al inicio va cambiando gradualmente a través de los videos, llegando a una dinámica horizontal en la que todas las personas tienen la oportunidad de proponer e invitar al movimiento. Las personas comienzan a decir desde sus propias experiencias traducidas a sus cuerpos.

\section{Discusión}

Hay una suerte de distribución de lo sensible (Ranciere, citado en Macías, 2009) que radica en la posibilidad dada solamente a las clases sociales altas de reflexionar sobre sus propias vidas, además de un régimen de lo sensible, en el que los grupos poderosos indican a las clases bajas qué deben sentir durante experiencias estéticas. En esta distribución, las personas que nacen en estratos menos privilegiados (clase bajas, de contextos rurales, bajo nivel educativo) no llegan a tener acceso a experiencias de reflexión personal y de inserción social desde lo corporal $y$, cuando las tienen, se les dan directrices de cómo comportarse, qué decir, qué hacer y qué sentir con respecto al arte. Este es el primer punto de ganancia en una intervención 


\section{Artículos}

desde la danza social en una comunidad costera, de bajos recursos económicos: se le dio a esta población la posibilidad de experimentar sus movimientos sin restricción de calificaciones o juicios de valor.

En este recorrido de sistematización y análisis se estudia el encuentro de una comunidad con un espacio para la reflexión desde sus propios cuerpos, espacio que se presenta con una consigna de libertad para explorar el movimiento. De lo recabado se pueden analizar dos niveles: en un primer nivel amplio se encuentra lo que podríamos describir como las consecuencias políticas de una propuesta de trabajo desde el cuerpo en la comunidad. En un segundo nivel más acotado, pero en relación íntima con el primer nivel, estaría la faceta más concreta de la intervención de danza social, y tiene que ver con las características de la metodología utilizada para incluir el cuerpo y el movimiento como motores de cambio personal y social. Es decir, un análisis de lo que dicha propuesta hace surgir en el contexto externo de la comunidad donde se lleva a cabo y un posterior análisis de lo acontecido hacia lo interno del proceso.

\section{De la Compañía bananera a la Compañía de danza}

Recapitulemos. De una llamada hecha en busca de una capacitación privada nace una intervención social cuya metodología es la danza y el movimiento, la cual viene a insertarse en un contexto en el que los cuerpos han sido localizados, colonizados históricamente por un microsistema político-económico que se denominó United Fruit Company. Este microsistema determina hasta el presente la posición social de los miembros de su comunidad, con base en el poder que dotan los puestos de trabajo dentro de la compañía, dejando una huella histórica que obedece a dicha clasificación, aun cuando el terreno en cuestión ya no está ocupado por la compañía, sino por una institución educativa democrática, en principio abierta a la comunidad.

Como se describió al inicio, la clasificación de las personas realizada por la UFCo -heredada hasta el presente- implica una colocación de los cuerpos que incide directamente en la identidad y experiencias de las personas. El espacio determina la vida de las personas porque, en principio, la colocación es concreta -funcional, geográfica y corporal-: es cierto que las personas pueden o no ingresar a ciertas áreas del territorio, pero siendo así, es política, y en última instancia es personal: el área a la que se puede ingresar determina la accesibilidad a oportunidades o falta de la misma.

Entrar en la universidad durante estos talleres significó romper con ese paradigma tan introyectado en las personas de la comunidad, dar cabida a la resistencia de los cuerpos en su excesiva fuerza. Este acto resulta sumamente importante para su identidad, ya que reivindica su derecho a acceder a la educación (a través del ingreso y reconocimiento de la Universidad) y a la cultura (a 
través de la participación en una actividad de creativa). Reivindica, de igual forma, su derecho de ocupar el espacio, mientras que diluye el sello que el espacio le imprime según su estatus.

Como segundo punto, los cuerpos que transgreden la prohibición del espacio, lo hacen descolocándose de lo que el espacio y la historia les heredó, creando movimiento y reflexionando desde su corporalidad, resignificando el espacio, las relaciones con otros y las ideas que habían tenido sobre sí mismos. Es un movimiento dentro del amplio movimiento de entrar donde antes tenían prohibido por su posición social.

En esta red de significados, la creación de movimiento es especialmente significativa para la comunidad porque les recuerda su poder para elegir dónde moverse, cómo moverse y con quién moverse. Como se observa en la entrevista realizada y en los videos, los talleres dejan atrás las etiquetas económicas y políticas, para ser cuerpos en movimiento, en contacto físico, creando una dinámica social que recuerda a estas personas el sentido de comunidad.

Lo anterior se concibe como un hecho de especial relevancia en una zona que se encasilla en el intercambio económico, ya que se resaltan las posibilidades de aliarse en actividades educativas y culturales, para que sus miembros se experimenten a sí mismos desde estas posibilidades también.

Partiendo de lo anterior, la pregunta esencial es: ¿qué hace importante a esta intervención desde la danza, en contraste con otros tipos de intervención? Realmente existen diversas metodologías que podrían también haber servido para el propósito de sanar esta herencia en la comunidad, pero la danza contemporánea tiene características especiales. En primer lugar, lo que la hace relevante es la literal y metafórica descolocación del cuerpo. Poder actuar esta descolocación-descolonización de sus cuerpos, para pasar a resignificar los espacios accesibles y, a través de ellos, las oportunidades accesibles en una forma que de manera estética dice algo como: "aquí estamos y ya no somos los que nos hicieron creer que éramos". Así, lo que la destaca, es su aporte a la simbolización de sus historias de vida desde y a través de sus cuerpos.

Encontraremos la disposición del sujeto que busca ya no la palabra directa, sino aquella experiencia de reelaboración de relaciones que han perdido frente al lenguaje, como una forma de la subjetividad contemplada desde su encarnación, una forma posible de la presencia propia y la relación con los otros. Como ejemplo de esta subjetividad encarnada, tendremos el caso de la danza, que claramente nos enfrenta a un sistema que siempre será más que una suma de signos e implicará una significación productora de sentidos siempre diferentes. (Macías, 2009, p. 112)

Para realizar esto, y entrando aquí en el segundo nivel de análisis, la metodología de la danza social debe tener ciertas características en la comunidad. En primer lugar, debió saber escuchar el contexto social y político de la zona; ser consciente de la ruptura que 


\section{Artículos}

provoca al ingresar al terreno universitario en este contexto e imaginario; conceder el espacio para que, corporalmente, la comunidad pueda sanar esta herencia histórica y leer detrás de las propuestas este pasado político que dejó huella en los participantes. Este es un aprendizaje importante: proponer las estrategias de intervención en danza con una previa investigación del contexto social. Mirar primero qué sucede en la comunidad para ajustar la metodología con respecto a esos contenidos simbólicos, con la claridad de que estos siempre se alojan en los cuerpos.

Esta riqueza simbólica debe aprovecharse en un proceso democrático de inserción en la comunidad, donde el movimiento nazca de las personas que participan del taller, no de la imposición de los cuerpos profesionales que vienen a dirigir la actividad. Si no se plantea de esta manera, se corre el gran peligro de hacer de la danza un nuevo dispositivo de poder y así volver a colonizar estos cuerpos, siempre desde una posición externa, en lugar de promover el intercambio y la creación desde las corporalidades autóctonas.

La libertad con que se pueda participar de las propuestas y también proponer desde lo propio, como se dio en este proceso a partir de proponer las temáticas, generar el movimiento e incluso solicitar la música a utilizar, por ejemplo, son elementos básicos que diferencian una intervención social liberadora de un dispositivo de poder más, como se ha visto muchas veces en procesos rígidos de formación en danza, que también colonizan la corporalidad de la persona danzante, silenciando los impulsos propios que esta persona quisiera expresar o elaborar desde su cuerpo.

En el caso de un proyecto de danza social, la creación debe fluir de manera más natural, permitiendo que se problematice la colocación de sus cuerpos, los dispositivos de poder que les atraviesan y creando conciencia en los mandatos sociales que les limitan su libertad para elegir. Para esto es fundamental que la persona que facilita el proceso tenga la capacidad de cuestionarse, y reflexione en torno a los dispositivos de poder que le atraviesan a sí misma. Tal como dice Macías (2009):

Si bien no se puede prescindir de los dispositivos formales, de la técnica de formación dancística ni del encuadre que visibiliza cierta realidad, la presencia, como un decir asomándose del cuerpo que surge a partir de la representación, siempre insinúa ese algo más excesivo que se manifiesta, no como reflejo sino como fuerza, expresión de la práctica que se ubica en la propia vida, es decir, en el cuerpo. (p.132)

La persona que facilita un contacto con el movimiento debe encontrar ese "algo más", que se libera del poder (incluso el poder de la danza) para no bloquear en las otras personas lo que emana de sus cuerpos, sino más bien promover que sigan creando cada quien a su modo, con las herramientas que tengan. El cuerpo será, en este caso, para cada uno su espacio de trabajo y reflexión, y cada quien deberá decidir si desea mostrar lo que reflexionó o si desea guardarlo para sí. De 
cualquier modo, la ganancia para sí mismos y para la comunidad está dada. Aquí es oportuno presentar la percepción de Fritz (en Le Breton, 2005) quien afirma que:

La danza contemporánea vendría a ser un «instrumento de conocimiento y lenguaje» que busca un diálogo fuera de los códigos establecidos. A diferencia del teatro, que da pistas más próximas a lo cotidiano, la danza contemporánea muestra el cuerpo liberado de códigos culturales, construyéndose ella misma en lo efímero de su gesto, en un cuerpo inédito. La danza es invención de forma y contenido. El cuerpo aparece más que el cuerpo; el mundo más que el mundo. (p.15)

Es decir, la danza rompe con los códigos establecidos socialmente para la corporalidad y se presenta como acción en la que estos se cuestionan y se propone, a su vez, una libertad de dichos códigos. La danza contemporánea es en sí misma una ruptura con los códigos establecidos para el cuerpo, lo cual es esencialmente político en todo contexto, $y$, para este contexto particular representa una liberación del investimento que el poder económico y político marcó.

Asumir la tarea de proponer formas de accionar al cuerpo como motor de nuevas relaciones, implica construir modos de sensibilidad y de relación con el otro, que se hagan cargo de la fragilidad de cualquier cuerpo no acabado (es decir, de cualquier cuerpo). El cuerpo vulnerable, partícipe de un espacio habitado, llenándose y llenando de significados, que siente al ser impactado, que percibe y utiliza los sentidos, haciendo que lo vivencial sea corporal y el mismo tiempo generador de relaciones desde el exceso y la gratuidad que lo define, abre su posibilidad como acontecimiento al engendrar formas que vislumbran el borde de algo más...posibilidad que en la danza contemporánea se hace manifiesta. (Macías, 2009, p. 106)

Es fundamental partir de que la danza, como experiencia corporal, no puede ser neutral; esta se ubica dentro de un medio social y y se encuentra atravesada por una historia personal y un medio sociopolítico del intérprete. Responde a ese sistema de valores y significados de alguna manera. Ubicar el cuerpo en lo público implica generar movimiento del status quo. En este sentido, aparte de ser un ente político, el cuerpo es, en la danza, un instrumento por el cual se habla, por el cual se establecen inquietudes. Es una forma de inscripción y cumple una función saludable en la historia personal de la persona danzante.

Los nuevos planteamientos del hacer dancístico, que no anuncian una nueva manera de moverse sino una experiencia no limitada a la mirada, convocan lejos de la percepción de la forma, y hacen su reto el plantear la intervención de un sujeto conscientemente corporal, que produzca corporalidades propositivas y que trastoque las relaciones entre el cuerpo y el entorno, más cercano a la producción de pensamiento que a la belleza y el hedonismo. (Macías, 2009, p. 134)

Procesos como el descrito en este trabajo dan solidez a la investigación de y desde los 


\section{Artículos}

cuerpos, para la transformación social desde el arte y las sinergias colectivas. Este trabajo busca establecer líneas de acción para la construcción de un bagaje teórico práctico que posicione la danza como dispositivo de resistencia y práctica de subjetivación. Además, es una invitación para el necesario desarrollo de un bagaje teórico desde y para la danza, aprovechando experiencias latinoamericanas, ricas en materia, desde el movimiento; para dejar atrás viejos vicios de investigación que no elabore teoría basada en la evidencia práctica, o bien, informes prácticos con gran contenido que se archiven, dejando de lado datos valiosos para crear conocimiento y compartirlo.

Es necesario que el contexto costarricense crezca como generador de conocimiento teórico en danza, que además de la línea de acción social de la Universidad con la que se cumplió a cabalidad en este proyecto, se apunte a una estrategia que convoque las líneas de docencia e investigación, posicionando al cuerpo, al movimiento y a la danza como fuentes fehacientes de conocimiento, capaces de intercambiar con otras áreas más desarrolladas del saber. La creación escénica en la práctica debe ir de la mano con un acervo teórico que le dé sustento y le dé un norte a su estrategia institucional y política.

\section{Referencias}

Jensen, H. (2012). Sintaxis del espacio y narrativa del poder: Arquitectura en Golfito. Reflexiones, 91 (1): 199-206.

Le Breton, A. (2012). Antropología del cuerpo y modernidad. Nueva Visión: Buenos Aires.

. (2015) El sentido del cuerpo. Entrevista publicada en la revista virtual Tendencias 21. Recuperado de http://www.tendencias21. net/David-Le-Breton-El-sentido-del-cuerpo_a69.html

Macías, Z. (2009) El poder silencioso de la experiencia corporal en la danza contemporánea. Artezblai: Bilbao.

Rodríguez, F. (2006). La estrategia socioespacial de las heterotopías: ¿el poder organiza espacios de exclusión o de fijación? Xeográfica, Revista de Xeografía, Territorio e Medio Ambiente, 6: 171-179. 